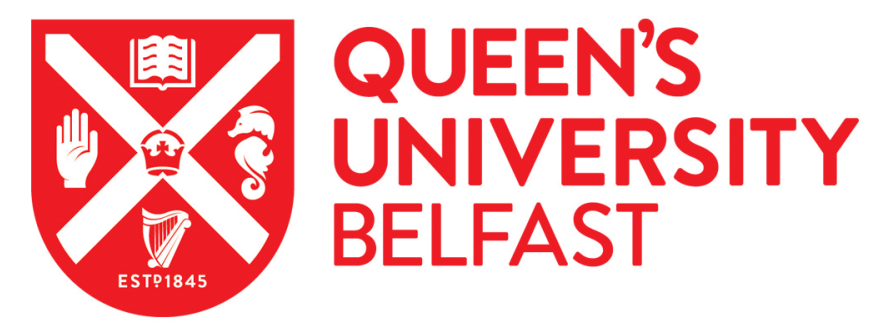

\title{
Educating Older Adults' Attention towards and Away from Gap- Specifying Information in a Virtual Road-Crossing Task
}

Stafford, J., \& Rodger, M. (2021). Educating Older Adults' Attention towards and Away from Gap-Specifying Information in a Virtual Road-Crossing Task. Ecological psychology, 33(1), 31.

https://doi.org/10.1080/10407413.2020.1826322

Published in:

Ecological psychology

Document Version:

Peer reviewed version

Queen's University Belfast - Research Portal:

Link to publication record in Queen's University Belfast Research Portal

\section{General rights}

Copyright for the publications made accessible via the Queen's University Belfast Research Portal is retained by the author(s) and / or other copyright owners and it is a condition of accessing these publications that users recognise and abide by the legal requirements associated with these rights.

Take down policy

The Research Portal is Queen's institutional repository that provides access to Queen's research output. Every effort has been made to ensure that content in the Research Portal does not infringe any person's rights, or applicable UK laws. If you discover content in the Research Portal that you believe breaches copyright or violates any law, please contact openaccess@qub.ac.uk. 
1

2

3

4

5

6

7

8

9

10

11

12

13

14

15

16

17

18

19

20

21

22

Educating older adults' attention toward and away from gap-specifying information in a virtual road-crossing task

James Stafford ${ }^{1}$, Matthew Rodger ${ }^{1}$

${ }^{1}$ School of Psychology, Queens University Belfast, David Keir Building, 18-30 Malone Road, Belfast, N.Ireland BT7 1NN, UK

18 Corresponding author: James Stafford

9 School of Psychology, Queens University Belfast, David Keir Building, 18-30 Malone Road, 20 Belfast, N.Ireland BT7 1NN, UK

$21 \quad$ Jstafford02@qub.ac.uk

22 Word count: 13564 (10329 excluding references and appendices) 


\section{Abstract}

24 Previous research has shown age-related declines in the use of specifying perceptual information to guide action decisions in traffic environments. In the present study, we investigated the effects of cross-modal cueing on perceptual training with older adults in a virtual road-crossing task. Specifically, we tested whether the visual information used to decide which inter-car gaps afforded crossing could be influenced by sound events which tracked either gap-specifying or non-specifying optic variables. Thirty-nine older adults were divided into three groups who practiced with auditory cues mapped to either the time-toarrival of the approaching car (specifying group), its distance (non-specifying group), or no sounds (control group). Although all three groups reduced decision errors with training, analysis of which variables predicted crossing responses showed that the specifying group's decisions became more attuned to the time-to-arrival information, whereas the non-specifying group became less attuned to this information and more to the distance information. Thus, attention for action decisions in older adults was re-educated toward either specifying or nonspecifying visual information, depending on the optic variables highlighted by the auditory cues. The implications of these findings are discussed in the context of designing perceptual learning studies and road safety interventions for the elderly. 


\section{Educating older adults' attention toward and away from gap-specifying} information in a virtual road-crossing task

Navigating safely through an environment of moving hazards is a gap-selection task. It requires one to perceive and select only those gaps between the hazards and oneself that will persist for at least the duration required to locomote through them. Crossing road traffic is such a scenario. To cross a road of moving vehicles safely, a pedestrian's decision to initiate action (walking) must ensure that the time left until any oncoming vehicle intersects her path of heading is greater than the time it will take her to pass that intersection point. Thus, in principle, successful action-decisions in dynamic environments like this should be based on prospective perceptual information that specifies spatial-temporal closure of relevant hazard-pathway gaps. However, while such information may be contained in the optic array from the perspective of the pedestrian, it is not clear that this information is universally the basis of road-crossing decisions. Indeed, some individuals may base decisions to cross on non-specifying information relating to the perceived distance or velocity of the approaching car. This is risky, as the persisting duration of a cross-able gap is not determined by either of these variables alone. This leads to the question of whether it is possible to train perceivers to tune into more 'temporal-gap-specifying' sources of information in the optic array as the basis for deciding whether and when to initiate action. To address this question, we here investigated the malleability of the perceptual information on which action-decisions in dynamic environments are based.

\section{Candidate information sources for gap selection}

In the scenario described above, a spatio-temporal gap between the oncoming car and the intersection of the pedestrian's heading which will persist for at least as long as the time 
taken for the pedestrian to pass that intersection point constitutes the affordance of roadcross-ability. The primary function of vision for this task is to detect the information in the flowing optic array which specifies this affordance. If the pedestrian is attuned to information which lawfully specifies crossable spatio-temporal gaps, such that this information guides their action decision and control, then they should enjoy a safe and successful road-crossing career. However, should they fail to detect such information, and/or guide their crossing decisions and behaviour based on features of the optic array which do not lawfully specify the prospective gap closure, then they risk injurious collision. Research has been directed at asking both whether there are informational invariants in the optic array which specify prospective gap closures, and whether humans guide actions using such information.

One candidate source of visual information for prospective spatio-temporal gap perception in the road-crossing situation described here is $\operatorname{tau}$ (Lee, 2009), which is the ratio between the magnitude of an optical solid angle and its current rate of change. For a directly approaching object, the ratio between the angular extension of the object's visible surface and this angle's rate of change specifies the time remaining until the object will collide with the point of observation. For two objects approaching each other in the visual field, the ratio between the angular extension of the closing gap between the two objects' surfaces and this angle's rate of reduction specifies the time remaining until the objects collide with each other. Thus, optic tau would appear a viable candidate for information specifying both the prospective time-to-closure of the gap between a moving car and a pedestrian's line of crossing, and the time-to-collision of the approaching car as they cross.

There is some scientific evidence that skilful actors may make use of optic tau for perceptually guiding spatio-temporal gap selection and action. In sporting scenarios which require players to negotiate spatio-temporal gaps between themselves and opponents and/or teammates, action-based decisions have been shown to be supported by attuning to tau-based 
information (see Watson et al., 2011; Correia, Araújo, Craig, \& Passos, 2011; Correia \& Araújo, 2010). However, a number of researchers have found that humans may also rely on optical variables that are not lawfully specific to the to-be-perceived time-to-contact of an event, but which are merely correlated with the environmental property, known as nonspecifying sources of information (Withagen, 2004). For instance, Smith, Flach, Dittman, and Stanard (2001) asked participants to release a pendulum at the appropriate time so that it would make contact with an approaching rolling ball at a fixed point on the ball's trajectory. Smith and colleagues pointed out several optical variables which observers might use to carry out this task, including angular expansion rate of the ball, which relates to the ball's velocity and is merely correlated with time-to-contact, or optical tau. Importantly, angular size and rate of expansion co-vary with the ball's size, and so are not invariant across different sized balls, while tau is. Over the course of practice at the task with balls of different size, participants shifted from use of non-specifying information (i.e. expansion rate) to greater use of specifying information (i.e. tau) to specify the affordance of intercept-ability. Gibson (1969) referred to this process of tuning towards more useful perceptual information for the specification of affordances as the education of attention.

\section{Training methods for educating attention in older adults}

The Smith et al. (2001) experiment provides evidence that even though there may be visual information available to specify the time-to-closure of a gap between a moving object and a point in the scene, some observers may fail to detect this information or guide actions on the basis of it. This idea is relevant to the current study, as the tendency to guide action through perception of non-specifying information in gap selection tasks may be more prevalent in older adults compared with other age groups. Ever since JJ Gibson (1979) formulated the concept of affordances, ecological psychologists have shown how younger, university-age adults are attuned to information specifying what actions can be performed in 
a variety of contexts such as passing the body through apertures (Franchak, Celano, \& Adolph, 2012; Warren \& Whang, 1987), standing upright on inclined surfaces (Fitzpatrick, Carello, Schmidt, \& Corey, 1994), fitting a hand into an aperture (Ishak, Adolph, \& Lin, 2008), and stepping across obstacles (Cornus, Montagne, \& Laurent, 1999). However, the picture regarding attunement and the elderly is less clear. Visual acuity and sensitivity to visual motion declines as the number of visual impairments and eye conditions increases with age (Owsley, 2011; Poulter \& Wann, 2013). These age-related changes may affect detection of dynamic affordances like cross-ability of a spatio-temporal gap between a moving object and one's line of travel.

Accordingly, educating the attention of older adults to different optic variables for prospective gap selection may require a method of highlighting the particular information in the participant's optic flow field during perceptual training. One method of training that has shown to be effective for perceptual learning in older adults is cross-modal cueing (e.g. Lees et al., 2012). When different properties of an event are perceptually accessible through multiple sensory modalities, event information can be detected concurrently ('supramodally') across the sense modalities to inform actions (Rosenblum, Dias, \& Dorsi, 2016). This appears to be especially relevant when unisensory information alone is unreliable (e.g. Ernst \& Banks, 2002). Given that ageing is known to be associated with both visual and auditory deterioration (Allard, Lagacé-Nadon, \& Faubert, 2013), it has been suggested that older adults may derive greater benefit under training conditions which combine information across visual and auditory modalities. Indeed, auditory cues can be designed to highlight task-specific information, rather than altering the task in terms of the perceptual information that supports action decision and control (Dyer, Stapleton, \& Rodger, 2017). In contrast, the presence of augmented visual cues (e.g. providing a coloured sphere that guides learner's attention towards higher-order optic variables; Lynch, Olivier, Bideau, \& Kulpa, 2019) may 
result in learning being tied to the presence of this information and may not generalise beyond these conditions (i.e. when augmented feedback is removed from the natural task setting).

This phenomenon of learners relying too heavily on the augmented information during practice and showing a significant decline in performance when this information is removed is known as the 'guidance hypothesis' (Park, Shea, \& Wright, 2000). In the present experiment, augmented auditory cues were presented to participants during training in an attempt to ameliorate guidance effects by educating visual attention to relevant co-occurring optical information that would be present in the scenario anyway. For instance, similar to the visual domain, there is evidence that time-to-arrival (TTA) judgements can be specified by a tau-like ratio of the object's instantaneous acoustic intensity to its instantaneous rate of change (Shaw, McGowan, \& Turvey, 1991). This is often referred to as auditory tau. If the relevant TTA information does take on the same form in both sensory modalities, it is possible that action-decisions can be formed in a manner that is agonistic about sensory modality (Gordon \& Rosenblum, 2005). Indeed, recent research has shown that when both visual and auditory cues are available to an observer, TTA judgements incorporate both auditory and visual information (DeLucia, Preddy, \& Oberfeld, 2016; Keshavarz, Campos, DeLucia, \& Oberfeld, 2017). Interestingly, however, when visual information is removed, both younger and older adults appear to rely more on non-specifying sources of auditory information (e.g. the sound pressure of the final auditory cue; Keshavarz et al., 2017). As a result, cross-modal cues may be able to educate older adult's attention both towards and away from gap specifying information, depending on the specifics of what information the two modalities actually have in common.

Another process which likely supports perceptual learning is attention to the mapping between information variables used to guide action and success or failure of the 
corresponding task outcome (Jacobs \& Michaels, 2007). After a period of practice, perception improves because observers learn that variables that were initially relied on before practice do not produce consistently accurate judgements. However, if the initial nonspecifying variables were not rendered useless during practice then the observer may continue to use those variables to inform action decisions (Jacobs, Runeson, Michaels, 2001). Non-specifying variables may continue to guide actions either because they yield reasonably accurate performance over the course of practice or because the perceiver did not learn that the attended variable was non-optimal. To address the former, practice conditions should be organized so the use of a non-specifying variable will result in repeated misjudgements of what the environment afforded. To address the latter, observers must have access to knowledge of the results of a practice attempt. However, during our everyday life such knowledge of results are often unavailable to us. For example, when we decide to reject a gap between moving pedestrians in a crowded airport we do not know for certain whether that decision was right or wrong and to what extent. To avoid overlapping utility of specifying and non-specifying information, the present experiment designed conditions so that two nonspecifying variables (gap magnitude and gap closure-rate) resulted in a low percentage of successful decisions. Furthermore, participants were provided with knowledge of results (KR) feedback to allow participants to compare their judgements to previous attempts during practice.

\section{Training older adult's to select gaps between moving vehicles}

Perceiving affordances is usually much more complex when objects are moving than when they are stationary. This is largely due to the fact that available affordances in these environments change over time, coming into existence and then dissipating as objects continue to move relative to each other and the actor. Research in road-crossing behaviour has found that older adults regularly base their decisions on whether to cross or not by 
waiting until an inter-vehicle gap reaches a certain distance (corresponding to an opticalangle-size strategy) rather than a prospective time-based strategy that incorporates both the optical size of a vehicle and it's rate of expansion (Lobjois \& Cavallo, 2007; Oxley, Ihsen, Fildes, Charlton, \& Day, 2005). Such a finding is reinforced by research showing reduced sensitivity to the rate of expansion of an object in the retina (often used to gauge an approaching vehicle's speed) with increasing age (Poulter \& Wann, 2013). In spite of identifying this age-related difficulty, behavioural interventions have so far failed to successfully attune older adults to specifying sources of information (Coeugnet et al., 2017; Dommes \& Cavallo, 2012; Dommes, Cavallo, Vienne, \& Aillerie, 2012). For instance, Dommes et al. (2012) designed a road-crossing training protocol using virtual environment technology in which older adults were encouraged to assess the approaching vehicle's speed before crossing, rather than considering its distance only. The authors found that instead of abandoning a distance-based strategy, participants shifted where they perceived the actionboundary or "critical distance" to be, i.e. they continued to base action decisions on a nonspecifying optical variable, albeit as a modified function of that variable. Thus, it remains an open question as to whether training can induce tuning between the available optical information variables to perceptually guide gap-based affordance decisions and actions.

Crucially, the above-discussed training approaches have several limitations. First, explicit instructions to attend to an approaching vehicle's "speed" fail to educate the elderly pedestrian's attention towards information in the optic flow field that guides decisions about when and how to act. Rather, they highlight third-person physical variables that are not defined relative to the perceiver at the ecological scale. Recent research has shown that the optical variable tau can be used to perceive the affordance of an inter-vehicle gap without the need for prior knowledge of speed or distance of the approaching vehicles (Stafford, Whyatt, \& Craig, 2019). Furthermore, in previous perceptual training studies, distance information 
could support reasonably accurate performance before and after training with participants showing behavioural improvements despite still not attuning to the vehicle's speed. As optical expansion is correlated with optical tau, it may have adaptive value for deciding whether or not to cross the road (i.e. it will sometimes, but not always specify passability). Therefore, these training paradigms reward participants for changing the way that the nonspecifying variable they were already attuned to is used to determine an opportunity for action, rather than judgements only being systematically rewarded when switching to a more useful variable.

Finally, it is unclear whether highlighting one source of non-specifying information (i.e. rate of optical expansion) to an individual who is already attuned to another source of non-specifying information (i.e. optical size) will help guide a learner to a specifying sources of information (i.e. optical tau). The present work aims to investigate if perceptual training which emphasises one or other optical variable in the visual scene will induce a shift towards use of that variable in subsequent action decisions. Accordingly, three candidate information variables will be considered: time differential, gap magnitude, and gap closure-rate. The time differential variable is considered to be specifying as it encompasses the time to closure of a motion gap between the pedestrian's line of travel and the approaching vehicle at its current rate of closure (Bootsma \& Oudejans, 1993). As previous research has shown older adults tune in less to this time-based variable when making action decisions in traffic environments (Stafford et al., 2019), it is of paramount importance to understand if this age group can relearn to use this information. In contrast, both gap magnitude (i.e. vehicle distance) and gap closure-rate (i.e. vehicle speed) are considered to be non-specifying as they are confounded by factors unrelated to TTA such as object size (van der Meer, van der Weel, \& Lee, 1994). As older adults have been shown to rely on to gap magnitude when selecting inter-vehicle gaps (e.g. Lobjois \& Cavallo, 2007) and previous training studies have aimed to highlight gap 
240 closure-rate information (e.g. Dommes \& Cavallo, 2012), we were interested in how sensitivity to these lower-order variables change when they are either highlighted or deemphasised via cross-modal cues.

The aim of the present work was to further understand the effects of cross-modal cues on visually-guided action decision-making in older adults. Three groups of older adults participated in a perceptual training study based on a virtual road-crossing scenario. They had to view a traffic scene in a virtual reality (VR) environment (from the first-person perspective of the virtual pedestrian) and decide whether (and if so, when) to initiate a road-crossing walk between two vehicles (a leading and a tailing car, which together formed a transient spatiotemporal gap). Car speeds and distances were varied across trials, and once initiated, the duration of the actual road-cross was fixed for all trials. Fixing the road-crossing speed of the virtual pedestrian served to both standardise the execution of the selectable action, and to determine which gaps were or were not safe to select. All participants received feedback in training about whether their selection and timing was accurate (i.e. safe cross or collision). Furthermore, two of the three groups heard a series of discrete increasing-intensity sound cues in each trial that either mapped onto the decreasing time-to-arrival of the tailing car (specifying group), or mapped onto its decreasing distance to arrival (non-specifying group). A control group heard no sound cues during training. To test whether the mapping of sound cues to either specifying or non-specifying optical variables during training had an effect of educating attention towards the respective optical variable, we compared the groups before and after training in terms of changes in task performance and the predictive power of each variable on action selection. 
Participants.

Thirty-nine older adults aged 61-82 (26 female, 13 male) participated in the study.

One participant was removed after screening for symptoms related to simulator sickness before being assigned a group (see materials section). They were randomly allocated to three experimental groups of equal size $(n=13)$ : specifying group $\left(M_{\text {age }}=68.85\right.$ years, $S D=6.1$ years); non-specifying group $\left(M_{\mathrm{age}}=71.69\right.$ years, $S D=7.8$ years $)$; control group $\left(M_{\mathrm{age}}=\right.$ 70.38 years, $S D=6.4$ years). Although all participants reported having normal or correctedto-normal vision, $30.77 \%$ of the specifying group, $23.08 \%$ of the non-specifying group, and $30.77 \%$ of the control group still scored below average on a Snellen test for visual acuity (lower than 20/25) while wearing their corrective lenses. However, all participants were able to detect the presence of the oncoming vehicle in virtual reality from its furthest position. None of the participants required hearing aids for the study and all reported they could hear the quietest sounds used in the experiment $(-21 \mathrm{~dB}$ relative to the maximum output of the Oculus Rift headset). Queens University Belfast ethics committee granted ethical approval, and all participants gave written consent before testing began. Participants were naïve with respect to the scientific hypothesis of the study.

Materials.

The simulator sickness questionnaire was completed before testing began to ensure the participant was not at an increased risk of sickness while wearing the VR headset for extended periods of time (Kennedy, Lane, Berbaum, \& Lilienthal, 1993). The questionnaire assessed each participant's history of motion sickness, claustrophobia, dizziness, nausea and migraines. Any participant reported these symptoms prior or during testing was excluded $(n=$ 1). The virtual environment (representing a traffic-filled road) was presented in an Oculus 
view of 100 degrees, and updated 90 times per second. Real-time tracking of the head orientation while navigating through the virtual environment was achieved using the Oculus Sensor which was placed in clear line of sight of the participant. To initiate crossing in the environment, the participant pressed the ' $\mathrm{A}$ ' button on an Xbox One controller connected to the same PC computer which ran the VR simulation and recorded participants' response data.

Design.

The virtual scene consisted of a two-lane road, 5.6 metres wide from sidewalk to sidewalk. Every trial included bi-directional traffic with two cars of different colours in each lane (a blue leading car and a red trailing car). Each lane of traffic was symmetrically aligned (i.e. cars in the near and far lane will arrive at the pedestrian's line of travel at the same time) to ensure only one gap was presented to the participant in each trial.

\section{$* * * * * * * * * * * * \operatorname{INSERT~FIGURE~} 1 * * * * * * * *$}

The task consisted of a combination of 18 unique speeds and distances producing 18 different time gaps (times-to-arrival) to cross the road (see Table 1). Conditions were designed such that information relating to either the tail car's distance gap, or this gap's rateof-change, were not reliable cues for safe/unsafe gaps by themselves across multiple trials, due to variation in these parameters across trials. The movement speed of the participant in the virtual environment was fixed at $1.42 \mathrm{~m} / \mathrm{s}$ reflecting the walking speed of an elderly pedestrian at a crosswalk (Coffin and Morrall, 1995). Additionally, the approaching vehicles' speed was constant for the duration of the trial. Given the width of the virtual road (5.6 metres), the time to cross the road was fixed at 3.94 seconds, the combination of walking speed and the TTA of the cars resulting in 50\% of the gaps presented affording safe passage and $50 \%$ of the gaps affording collision with the tail (red) vehicle. 
The experiment consisted of a practice block, pre-test, two training blocks with

feedback, a post-test and a retention test which were divided into three stages and carried out on separate days. The first stage consisted of a familiarisation period and practice block followed by a pre-test and finally, the first training block. The second stage consisted of the second training block followed by a post-test. These stages were carried out on consecutive days. The retention test was conducted to test whether any induced changes across the three groups were relatively permanent. This test was carried out 9 days after the post-test.

Feedback and cues for perceptual learning. feedback for both rejected and accepted gaps. When a participant rejected a gap, they would receive augmented KR feedback in the virtual environment in the form of an ' $\mathrm{X}$ ' or a 'tick', indicating whether their response was correct (see Figure 2). Furthermore, when a gap was accepted, augmented KR feedback was presented in the form of a safety margin gauge (Figure 2) indicating where the tail vehicle was with respect to the participant at the moment they reached the opposite curb. This was calculated by taking the time remaining until the tail vehicle reached the pedestrian's line of travel (specifying group) or the distance of the front bumper of the tail vehicle to the pedestrian's line of travel (non-specifying group). The control group also received KR feedback based on the time remaining but their gauge did not include any units on the $\mathrm{x}$-axis to prevent encouraging participants to scale their judgements to a particular information source. When a participant successfully avoided collision with the tail vehicle, the safety margin expressed on the right-hand side of the gauge was green, indicating a safe judgement. In contrast, when the tail vehicle collided with the participant before the opposite curb was reached, the safety margin expressed on the left-hand side of the gauge was red indicating an unsafe judgement. 

to either the tail vehicle's time-to-arrival to the intersection point (specifying group) or distance from the intersection point (non-specifying group). Auditory cues consisted of isochronous repetitions of a pre-recorded sound of a woodblock being struck; digitally increasing iteratively towards the maximum sound intensity of the playback by two decibels (from -21 to $-1 \mathrm{~dB}$, relative to the maximum output of the Oculus Rift headset, over 10 repetitions). This sound type was chosen to have a very clear temporal onset (attack phase of the amplitude envelope). For the specifying group, the auditory cues (consecutive sound playbacks) were triggered when the time-to-arrival (seconds) of the tail vehicle corresponded to the ranking of intensity of sound $\left(\mathrm{e} . \mathrm{g} . \mathrm{TTA}=10 \mathrm{~s}\right.$, sound intensity $=10^{\text {th }}$ loudest $(-21 \mathrm{~dB})$, TTA $=9 \mathrm{~s}$, sound intensity $=9^{\text {th }}$ loudest $(-19 \mathrm{~dB})$, etc. $)$. In contrast, the non-specifying cues were triggered when the virtual distance (in metres) of the tail vehicle corresponded to the ranking of intensity of sound (e.g. distance $=100 \mathrm{M}$, sound intensity $=10^{\text {th }}$ loudest $(-21 \mathrm{~dB})$, distance $=90 \mathrm{M}$, sound intensity $=9^{\text {th }}$ loudest $(-19 \mathrm{~dB})$, etc. $)$. As a result, the specifying auditory cues had a lawful relationship to optical information specifying the time remaining until the pedestrian-vehicle gap (car-gap) closed at the observation point of the participant. For instance, Figure 3 shows how, due to consecutive sounds' onsets mapping onto interval changes in the ratio of the car's optical angle relative to its angular rate of change, the specifying auditory cues relate one-to-one with optical tau. In contrast, the distance-based, non-specifying auditory cues coincide with interval changes in non-specifying optical information which are variant across different car speeds. Therefore, the distance-based auditory cues track a changing pattern in the optic flow field that is not invariant in relation to 
time-to-arrival cross different transformations in speed and distance and hence cannot singularly specify the 'crossability' of a gap (see Figure 3).

\section{***INSERT FIGURE $3 * * * * * * *$}

Procedure.

As the virtual environment was presented in a portable head-mounted display and headphones, it ensured all participants received the same visual and auditory experience despite the physical location of testing. As a result, older adults were given a choice of testing in a laboratory on campus or at a home visit.

At the first phase, once consent was given, participants completed the visual acuity test and the auditory detection task. Next, participants were asked to put on the HMD and sit in a chair within the tracked space of the Oculus sensor. A familiarisation period allowed the participants to get familiar with the virtual environment by turning their head to look around and get used to both the HMD and the feeling of being immersed in the environment.

Participants were instructed to initiate and view the automated road-cross 5 times without any oncoming cars to familiarise themselves with the movement speed of the simulator. Next, participants received the practice block consisting of 18 randomly presented trials encompassing all speed and distance combinations faced in the virtual environment resulting in 9 crossable gaps and 9 not crossable gaps. Participants were instructed to press the button to initiate crossing when the space between vehicles is big enough to cross or else wait until the next trial begins. After the practice block, each subsequent block (pre-test, training 1, training 2, post-test, retention) consisted of 54 trials, giving rise to a total of 270 trials. In between blocks, participants were offered breaks and refreshments by removing the headset. Within blocks, the 18 specific distance-speed-TTA combinations were presented separately three times and the order of presentation was randomized. The two auditory training groups 
384

received additional instructions from the experimenter, stating that the red (tail) vehicle will be generating sound information with the sounds getting louder as it comes closer.

Participants were instructed to factor in these sounds when making road-crossing judgements, but were not given any information about what the sounds meant, i.e. what variables in the visual scene they were mapped onto.

\section{Measures \& Data Analysis.}

For each trial, measures were calculated from the time series positional data in the VR simulation of (a) the participant waiting at the curb, (b) the lead vehicle that opens the gap, and (c) the tail vehicle that closes the gap. In accordance with the task instructions, within each trial, the time period considered relevant for decisions was the moment the lead vehicle's rear bumper passed the participant's curb position and the gap between the tail vehicle and the participant began to close. Closing gaps and events were calculated from motion in two perpendicular axes in the VR environment: the X-axis (parallel with the road car motion was in this axis); the z-axis (perpendicular to the road - participant cross motion was in this axis). Trials with safe / un-safe gaps, were determined using the difference in time-to-arrival values between the crossing gap (in z-axis between participant and the other side of the road) and the car gap (in $\mathrm{x}$-axis between the participant and the front bumper of the tail vehicle; see Figure 4). Two main features of participants' performance were measured: accuracy of gap selection, i.e. to what extent participants opted to cross during safe gaps and rejected unsafe crosses; perceptual information guiding decisions, i.e. which variables in the gap-closing scene predicted participants' likelihood to select or reject a gap for crossing. 
Two types of error rate were calculated to determine accuracy of gap selection. Safe errors were calculated as the percentage of trials in which participants rejected a gap that would have been safe to cross $\left(T T A_{\text {CarGap }}>T T A_{\text {CrossGap }}\right)$, while unsafe errors were calculated as the percentage of trials in which participants chose to accept a gap that afforded too little time to cross $\left(T T A_{\text {CarGap }} \leq T T A_{\text {CrossGap }}\right)$. As these types of error are not behaviourally equivalent (i.e. unsafe gap acceptance has far greater consequences than safe gap rejections), these two error types were analysed separately.

\section{***INSERT FIGURE 4***}

\section{Information variables predicting gap selection}

We next considered the following candidate information variables as predictors for participants' decisions to cross or not: time differential, gap magnitude, gap closure-rate. Note that although the calculations of informational variables were derived from a thirdperson perspective, i.e. in terms of positions and motions of the objects and observer in VR coordinate axes, it was assumed based on the geometry of ecological optics (e.g. Lee, 1998) that these variables in the looming optic array accessible to the observer correspond with the cars' motions towards the point of observation. This assumption was verified from recordings taken in the VR set-up prior to testing (see Figure 3 for this information in relation to optic tau and the auditory cues). Time differential was calculated as the difference between the (fixed) TTA of the automated participant road-cross walk and the TTA of the tail car when the lead car passed the participants' line of crossing (see Figure 4). Note that the approaching tail car was visible in each trial prior to the moment of the lead car's passing, so information specifying its TTA at this moment was available for detection. Gap magnitude was calculated as the distance between the tail car and the point of crossing in the $\mathrm{x}$-axis at the frame the lead car passed the participant. Gap closure-rate was the velocity of the car closing the gap 
between itself and the pedestrian's line of travel along the x-axis and was constant within a trial (although variable between trials). As the vehicles in both the near and far lane arrived at the pedestrian's line of travel at the same time, gap closure-rate was consistent regardless of which lane the participant was looking at.

Although the auditory cues in the non-specifying training conditions mapped onto the distance of the car, the fact that the isochronous sounds progressed at equally-spaced distance intervals entailed that these sounds tracked both distance (at individual sound onsets) and velocity (shorter interval durations between sound onsets in faster velocity trials). For these reasons, both distance and velocity were measured as potential predictors for road-crossing decision rates, as participants in this group may have been educated to one or other of these sources of information by the auditory cues.

As each informational variable (i.e. time differential, gap magnitude, and gap closurerate) has a unique value across the 18 conditions, they each provided a continuous scale by which rates of responses (cross/stay) could be logistically fitted. If participants were using temporal information specifying the crossability of the car-gap, it would be expected that crosses would occur more frequently for the longer temporal gap and less frequently for the shorter temporal gaps. Similarly, if gap magnitude information was being attuned to, participants would cross more often when the tail vehicle was at a greater distance from the observer and less often when this distance was smaller. Finally, if participants were using gap closure-rate information, participants would cross more often when the speed of the tail vehicle was slower and cross less often when this speed was faster.

\section{Statistical Analyses.}

To compare gap selection differences due to training and between the three groups, intra-individual means of safe (i.e. unnecessarily rejected), and unsafe errors were submitted 
to separate analyses of variance (ANOVA) with repeated measures on test phase (pre-test ,post-test, retention). For all ANOVA's, Greenhouse-Geisser adjustments for the degrees of freedom are reported in cases where the assumption of sphericity was not met. Post-hoc comparisons were conducted with Tukey's HSD test $(P<0.05)$.

To examine group differences in sensitivity to invariant information, and thus degree of attunement from the different auditory cues, the relationships between gap selection and the candidate informational variables was assessed. Onto each dataset, the best possible logistic function was fitted, represented by following the equation:

$$
y=\frac{1}{1+e^{-k(c-t)}}
$$

In this equation, $t$ represents the values of the candidate information source, $\mathrm{k}$ is the slope or steepness of the curve at point $c$, which is the value in which the participant switched from rejected crossings to accepted crossings (see Oudejans, Michaels, Bakker, \& Dolne, 1996). The $r^{2}$ values yielded by the logistic function are determination coefficients indicating the fit of the curve to the data. The critical value $(\mathrm{CV})$, variable $c$, where $50 \%$ of the responses are judged as cross was also calculated. If pedestrians were tuning into the time differential between the car gap and the crossing gap, this CV value should be close to or slightly above 0 . As the present experiment was designed as a training study, to ensure task difficulty most of the inter-vehicle gaps have been designed to be close to this critical value (see Table 1). Furthermore, the slope of the curve between $40 \%$ and $60 \%$ was examined. This value indicates how rapid the switch between one response (cross) and the other (not cross) about the critical value. A steeper gradient suggests a more rapid switch and a flatter gradient suggests a slower switch from crossing to not crossing indicating a greater range of uncertain judgements. In addition, a logistic function was fitted to each individual participant to allow an inferential analysis between groups. An analysis of covariance (ANCOVA) was 
conducted to study if post-training and retention attunement measurements covaried with the pre-test measurement in the experimental and control groups.

\section{Results}

Gap selection accuracy: did the auditory cues influence performance in the virtual environment?

To investigate if the auditory cues influenced the nature of the incorrect decisions, errors were divided into two categories: unsafe and safe errors (rates of errors are shown in Table 2.). Unsafe errors were defined as the percentage of the accepted inter-vehicle gaps resulting in collision due to the tail vehicle reaching the participant before the cross was completed. In contrast, safe errors were defined as the percentage of trials in which the participant missed an opportunity to cross due to the time-to-cross between sidewalks being less than the time-to-arrival of the tail vehicle. A repeated measures ANOVA comparing unsafe error rate between test phases (pre-test, post-test, retention test) as a within factor and training groups (specifying, non-specifying, control) as between factor revealed no significant main effect of training condition $\left(F(1,36)=.235, p=.792, \eta_{\mathrm{p}}^{2}=.001\right)$, test phase $(F(2,72)=$ $\left..186, p=.830, \eta_{\mathrm{p}}{ }^{2}=.02\right)$ or significant interaction between factors $(F(2,72)=1.974, p=.108$ $\left.\eta_{\mathrm{p}}{ }^{2}=.05\right)$. The rate of collisions was not affected by sound cue type nor knowledge-of-results feedback during training.

To compare changes in safe errors rates (rejected gaps that could have been safely crossed), a repeated measures ANOVA with Test Phase (within factor) and Training Group (between factor) was conducted on this measure. A significant main effect of test phase ( $F$ $\left.(1.549,55.761)=15.528, p=<.001, \eta_{\mathrm{p}}^{2}=.30\right)$ was found indicating that the percentage of missed opportunities decreased between the test phases. Post hoc tests (Tukey-Kramer) demonstrated significant differences between the pre-test and the post-test $(p=.001)$ and 
503 between the pre-test and the retention test $(p<.001)$. No significant difference was found

504

505

506

507 between the post-test and the retention test suggesting the effects of the training blocks on reducing safe errors were relatively lasting. However, the absence of a significant interaction between Training Group and Test Phase $\left(F(3.098,55.761)=.540, p=.662, \eta_{\mathrm{p}}{ }^{2}=.03\right)$ showed that the differences in response accuracy across the different test phases were not significantly affected by the type (or presence) of auditory sound cues presented in training. Furthermore, no significant main effect of auditory cue group was found $(F(1,36)=.550, p=$ $\left..582, \eta_{\mathrm{p}}{ }^{2}=.02\right)$ suggesting no overall group differences in the number of safe errors between the groups.

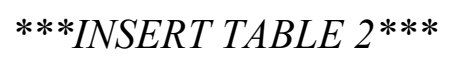
perceptual invariants?

To investigate if participants' attunement to different optical variables for selecting crossable road gaps varied with practice and/or between different types of auditory cueing in training, each group's crossing behaviour (i.e. percentage accepted or rejected crosses) was fitted as functions of time differential values, gap magnitudes, and gap closure-rates separately. Figure 5 shows these fitted functions for the time differential and gap magnitude variables. Due to gap closure-rate explaining a minimal percentage of the variance across each group and condition, we have moved this analysis to the Supplementary Materials section. The changes in information use across the three different test phases for each group are presented in Table 3 . 
non-specifying group, and 59.2\% in the control group. Each of the three groups demonstrated similar slope values suggesting a relatively gradual switch between stay and cross responses, as would be expected in the pre-test condition as none of the groups had received training to help attune to higher-order information sources (see Table 3). Additionally, the critical values presented in Table 3 indicate this switch occurred at similar time difference value for each group (between 0.32 and 0.37 ).

While the training did not visibly appear to change the informational variable participants most relied on in terms of the fitted function lines, the different forms of training did influence the extent to which participants relied on specifying information. In the posttest phase, Figure 5c shows that tau explained the most variance in the specifying and control group, explaining $81.2 \%$ and $80.8 \%$ of the variance respectively, which had both increased relative to the pre-test. For the non-specifying group, tau explained a reduced $47.2 \%$ of the variance indicating that the auditory cues were educating the group's attention away from perceptual invariants in the virtual road-crossing task. In contrast, Figure $5 \mathrm{~d}$ shows that variance in gap selection accounted for by the magnitude of the gap dropped to near 0 for both the specifying group, and the control group, while only decreasing to $36.9 \%$ for the nonspecifying group. In line with this, the non-specifying group also had the lowest slope value indicating worse discrimination certainty between cross and stay decisions (0.87).

Finally, the retention test showed that the effects of training were temporary or longlasting depending on the type of auditory of cues (specifying or non-specifying). Figure 5e shows that the time differential variable continued to explain the most variance, explaining $74.9 \%$ of the variance in the specifying group and $81.4 \%$ in the control group. For the nonspecifying group, variance explained by time-based variable increased back up to $58.1 \%$. Furthermore, both the specifying and control group retained their improved ability to rapidly 
551 switch between cross and stay judgements with a slope value of 0.6 whereas the nonspecifying group remained the least certain of their judgements (0.87).

Following the preceding analysis of the logistic fit between different variables which can be optically specified in the road scene and participants' crossing decisions at the group/test-phase level, we separately analysed logistic fits for each participant to allow inferential statistical comparison of the three groups based on the aggregated summary values of these fits. Each participant's rate of accepted cross was fitted as a function of the 18 time differential values and gap magnitudes (in separate fitting calculations) and $r^{2}$ values calculated from these.

An exploratory analysis comparing post-test variance explained by the time differential variable between the three training groups in post-test was conducted using a oneway ANCOVA, with pre-test $\mathrm{r}^{2}$ values as covariate. A significant main effect of group was found $\left(F(3,39)=4.115, p=.025, \eta^{2}=.19\right)$. Post-hoc comparisons using the Bonferroni test indicated that the variance explained after training by the time-based variable for the specifying group was significantly higher than the non-specifying group $(p=.022)$. No significant differences were revealed between the control and specifying $(p=.253)$ or the control and non-specifying $(p=.929)$. To investigate if the changes between groups were lasting, a one-way ANCOVA was conducted with the three auditory cue groups as the independent variable, the individual $r^{2}$ values by the time-based variable in the retention

571 phase as the dependent variable, again with pre-test $r^{2}$ values as the covariate. No significant main effect of group was found $\left(F(3,39)=1.883, p=.167, \eta^{2}=.10\right)$. 
575 training group (with pre-test as covariate) was found at post-test $(F(3,39)=2.130, p=.134$,

$\left.576 \eta^{2}=.11\right)$, nor at retention $\left(F(3,39)=.408, p=.668, \eta^{2}=.02\right)$.

577

578

579

580

581

582

583

584

585

586

587

588

589

590

591

592

593

594

595

596

597

598

\section{Discussion}

\section{Summary of findings}

The present experiment was carried out to test whether cross-modal events can influence perceptual learning in older adults. A VR road-crossing scenario was used to create an immersive optic scene, with the spatio-temporal gaps between cars forming transient affordances for crossing, relative to the fixed action capabilities of the observer (constant velocity motion across the road once a cross was initiated). Participants were split into different training groups who either heard auditory cues tracking the TTA of the approaching car (specifying group), the magnitude (or distance) of the car (non-specifying group), or no sounds (control group). All participants received summary (KR) feedback about the accuracy of their decision at the end of each trial. Results showed that older adults can improve in their visual affordance perception through training, and although cueing did not alter decision accuracy, it did lead to differences in the information on which participants based their decisions (specifying or non-specifying depending on cue types).

Participants in all groups improved after training in their abilities to accurately select crossable gaps. This was reflected in reduced rates for rejecting safe gaps, while the rate of selected unsafe gaps was unchanged by training. However, there were no main effects or interactions involving the group factor for accuracy rates, indicating that the presence of sound cues tracking different features of the visual scene did not influence gap selection behaviours. Moreover, the benefits in selection accuracy must be attributed to practice and/or the KR feedback provided during training (the lack of a no-feedback/no-cueing group prevents us from separating out the relative causal influences of these processes). 
Cueing features of the visual scene with auditory events did appear to have an effect on perceptual learning when we analysed the different variables in the scene which predicted participants likelihood to select a gap, comparing these before and after training. Participants

gap selection rate. Conversely, participants trained with sound cues tracking the non-

specifying (variant across trials) variable of the car's distance showed a reduction in fact that the control group's results most closely resembled that of the specifying group's suggests that the specifying cues may have been redundant in the context of this particular training scenario, with the greater effect being that of the non-specifying cues pull towards lower-order optical variables.

\section{Designing perceptual learning paradigms for older adults} information sources, suggests that cues that allow the use of multiple sensory modalities to perceive an event are not a prerequisite for perceptual learning in older adults. Rather, if practice is organized so that judgements based on non-specifying sources of information are systematically erroneous, older adults (with KR feedback) were able to determine the affordance of cross/not cross. This was achieved by selecting inter-vehicle gaps where the use of non-specifying sources resulted in collisions or missed opportunities and hence negative feedback in the simulator (either by colliding with the car and receiving a negative margin of safety or receiving a red ' $\mathrm{X}$ ' to signify a missed opportunity). This negative feedback associated with the use of lower-order variables appeared to be enough information to lead 
older adults to change the variable used and discover higher-order perceptual variables.

Indeed, such findings are consistent with the 'direct' approach to perceptual learning in which learners who initially rely on a variable that is made useless in practice come to rely on other more reliable variables (Jacobs, Michaels, \& Runeson, 2001; Jacobs \& Michaels, 2007). Importantly, these variables were already available in the optic flow field but the practice conditions encouraged the elderly participants to discover the need to use a more sophisticated time-based strategy to successfully navigate through the narrow inter-vehicle gaps.

An interesting question arises from the present results: if participants, as a result of training, are attuning to useful informational patterns to specify gap affordances, should we not expect corresponding changes in their behavioural responses? Importantly, increased sensitivity to specifying information about the environment does not necessarily lead to changes in behaviour. This is because the perception of affordances also requires a scaling to action capabilities i.e. perceptual-motor calibration (Brand \& de Oliveira, 2017; Jacobs \& Michaels, 2006). Indeed, previous research has shown how successful interceptive and avoidance actions are initiated at specific TTA values (Lee \& Reddish, 1981; Lee, Young, Reddish, Lough, \& Clayton, 1983). Thus, success in these tasks depends not only on detecting higher-order time information but also identifying what value or range of values of the informational available by which the action should be initiated. While the present study enabled the specifying and control group to converge onto more useful, time-based, information; participants may not have subsequently established the appropriate relation between the exploited informational variables and the fixed walking speed of the simulator. Interestingly, similar results have been found with child pedestrians who base their roadcrossing judgements on tau information to the same extent as adults but switch from an unsafe gap to a safe gap too soon indicating they are using a non-optimal tau value (Stafford 
et al., 2019). It must still be acknowledged, however, that the lack of group differences observed in task success might also reflect general difficulties in detecting TTA-specifying higher-order information among our sample of participants, or a lack of statistical power within this study to detect such an effect.

The performance measures in the present study reflect the difficulty in training both behavioural and perceptual adjustments in the older adult population in traffic environments. Despite the task not requiring older adults to physically cross the road, removing the need for self and object synchronization, and the specifying group receiving additional reliable auditory information, changes in behaviour were found across test phases for all groups, independent of the cross-modal cue condition. Similar challenges have been found in simulator-based behavioural training studies involving elderly pedestrians where training has influenced performance with older adults becoming more cautious and reducing the number of collisions, but failing to modify the perceptual strategies participant's adopt (e.g. Dommes \& Cavallo, 2012). In contrast, the present results showed, in general, that the auditory cues influenced the ability to choose temporal gaps based on higher-order information that specified the TTA of the approaching vehicle. The participants who benefitted most from the training intervention were the in the specifying group, which during the post-training phase selected gaps consistent with the use of tau information. In contrast, the non-specifying group's attention was directed away from the affordance-specifying invariants suggesting that cues which are potentially ambiguous with respect to environmental properties are not an effective method for augmenting perceptual learning, and may actually cause attunement to less reliable perceptual information. However, the retention test appeared to show that the effects of non-specifying cues were not long-lasting as no significant differences were found between groups in their ability to make decisions consistent with tau information. This may 
673 reflect intervening calibration due to real-world experience in the days between the post-

674 training and retention sessions.

675

676

677

678

679

680

681

682

683

684

685

686

687

688

689

690

691

692

693

694

695

696

\section{Applications of the present experiment for road-crossing interventions}

The present study has implications for the ongoing discussions around age-related degradation of TTA estimations and preventing unintended injury in traffic environments. Indeed, over the last two decades or so, there has been an upsurge in the number of studies investigating age-related decline in time-to-arrival estimations (Petzoldt, 2014; Butler, Lord, \& Fitzpatrick, 2016) and judgments about whether they are on a collision course (DeLucia, Bleckley, Meyer, \& Bush, 2003). However, recent research has suggested that the examination of attunement should be on the agenda in research involving the elderly, as aging appears to impact the capacity to attune to specifying information (Withagen \& Caljouw, 2011). As a result, it is important to investigate if elderly pedestrians can perceptually re-learn to judge affordances in dynamic environments. Crucially, the process of attunement is not only important for the present study but also a prerequisite to behave adaptively in the natural environment.

The need for more studies investigating perceptual attunement can be reinforced by earlier simulator-based studies that have found difficulty in improving elderly pedestrian's ability to take an oncoming car's speed into account (Coeugnet et al., 2017; Dommes \& Cavallo, 2012; Dommes et al., 2012). The authors of these studies have largely attributed difficulty in perceptual training to the fact participants, often voluntarily, decided not follow the advice delivered in training. Such advice could have been challenged because older adults failed to perceive how the verbal feedback related to how they made gap judgements. In other words, after training the participants still incorrectly perceived the affordance of passability in the traffic environment which could be attributed to the limited capacity to pick up 
697 perceptual information specifying the time-to-arrival of the oncoming vehicles. However, these studies did not examine how the training influenced which perceptual variables were used to detect this affordance, which can range from variables weakly correlated to the timeto-arrival of the oncoming car to sources of information highly correlated or even specific to time-to-arrival.

This is the first study to chart the changes in sensitivity to specifying information in a road-crossing training task. Of particular importance is the finding that older adults can relearn to use perceptual-based information to guide decisions about when to act. Previous work has cast doubt on whether this ability can be trained due to age-related visual decline and reliance on non-specifying information post behavioural training (Dommes \& Cavallo, 2012; Dommes et al., 2012; Poulter \& Wann, 2013). The present experiment has shown that when training is designed in such a way to reward the pickup and use of a perceptual variable that specifies the time-to-arrival of an approaching vehicle, an older adult can tune into this variable successfully. Furthermore, the present study emphasises that cues to direct attention towards specifying information sources must be lawfully related to that information source. It is not enough and can even be detrimental to identify an information source that participants are not attuning to (i.e. speed information) and then highlight this information alone. If augmented cues are to be introduced, they must ensure they relate one-to-one with the perceptual information they are highlighting in the optic flow field.

\section{Issues around event-to-information mapping}

This issue of event-to-information mapping in perceptual training entails consideration of some potential limitations to the conclusions that can be drawn from the present study. Firstly, although we adopt a model of event-to-optic specification in which the TTA of the car is mapped onto optic tau, and the distance of the approaching car is mapped 
onto the lower order variable of car angle magnitude (Lee, 1980), we cannot rule our other sources of information for these event properties in the optic array playing a role in participants' gap perception and decisions. For both our cueing approach and analyses, we assumed that these external dynamic properties (TTA / distance gap change) project onto the optic array of the viewer in VR (and indeed confirmed this for the auditory cue mapping, see Figure 2). This assumption does not rule out alternative explanations for how the car's TTA and distance might be optically specified. However, given that our sound cues were mapped according to these assumptions, any alternative explanations would need to account for how these cues would map onto alternative candidate informational variables to explain their effects.

The nature of the sounds used as cues, as well as their relation to the visual scene, need further consideration, development, and testing if this type of cross-modal education of attention is to be better understood. Although the consecutive sound events increased in intensity, this intensity change was not strictly mapped onto the distance of the approaching car squared according to acoustic laws (i.e. inversely proportional to the car's distance squared). Nor were other acoustic properties that vary with distance to source considered, such as attenuated power in higher frequencies with increasing distance (Farnell, 2010). Moreover, the event-based nature of the sounds did not directly correlate with any distinct event onsets in the visual scene, which was more continuous in how it unfolded. Thus, the use of sound cues to educate visual attention may be more effective if greater consideration is given to the source events in the environment about which the artificial auditory information is supposed to inform. Nevertheless, there were practical considerations at play in our current approach to sound cue design. Using changes across discrete sound events rather than continuously changing sounds (e.g. pitch-to-distance mapping) allowed us to differentially track higher- and lower-order variables in the visual scene while still being mapped to the car 
approach event, which would not be possible using a continuous sound type. There is work needed to resolve this tension between fidelity to source events and control over multi-modal augmentation of tasks/scenarios.

Finally, the lack of differences between the control group and the specifying cues group in information usage may have been influenced by the safety margin gauge presented during the two training. While no units were presented on the gauge to the control group during practice, the margin of safety was calculated using time remaining until the tail vehicle reached the pedestrian's line of travel. As a result, participants in the control group may have learned how the margin of safety was mapping onto TTA information and used this to detect higher-order variables. However, if this was indeed the case it would only account for judgements where the control group accepted the gap. As the augmented KR feedback for rejected gaps was the same for all participants ('tick' for correct, ' $\mathrm{X}$ ' for incorrect), an alternative explanation would be needed for why significant differences were found across test phases for the safe errors (where the KR feedback was the same) and not unsafe errors (where the KR feedback was different).

\section{Decoupling perception and action and ecological validity}

The picture regarding the training of older adult's road-crossing skills is also far from complete. It is important to note that our task involved only indicating if a gap afforded safe passage by pressing a button, separating the natural coupling between perception and action. Previous work has shown that when older adults are required to select and control their actions on foot, they delayed their initiation relative to the lead vehicle (Geraghty, Holland, \& Rochelle, 2016). The present experiment saw the participant sitting stationary on a chair delivering yes/no responses via a button press. Indeed, 'perception-only' go-no-go judgements tend to be less accurate than 'perception-action' judgements where the participant 
couples the perceptual-information to their movements (Cornus et al., 1999; Oudejans, Michaels, Van Dort, \& Frissen, 1996). Furthermore, Van Doorn, van der Kamp, de Wit, and Savelsbergh (2009) have revealed significant differences in gaze patterns between a perception-based task and an action-based task, suggesting that the detection of information is also impacted by whether participants are allowed to make judgements on the move.

Although the limited movement of participants in this study may have impacted on the results, Craig and colleagues (Craig, Bastin, \& Montagne, 2011; Craig et al., 2006) found when comparing goalkeeper's ability to judge a spinning ball's future arrival position in VR, that actors continued to use non-optimal sources of information, regardless of whether they were allowed to translate through the virtual environment. The authors argued that by using an HMD that allows the head to move and track an approaching object offers a significant improvement over traditional perception-only studies that use video images where the viewpoint is fixed to the camera angle and position and not to the observer's head position. This interpretation is supported by recent research which has found that even minimal head movements while wearing an HMD with a virtual environment that provides KR feedback can greatly improve estimations of time-to-arrival (Braly, 2020). Indeed, the concept of representative design (Brunsik, 1955) emphasises the need to have practice conditions that allow the observer to pick up perceptual information that specifies a property of the environment-actor system (Araújo, Davids, \& Serpa, 2005). It appears that the task constraints presented in the present experiment were able to maintain the sources of information required for performance to improve over practice. This is particularly relevant for road-crossing interventions aimed at older adults who may derive greater benefit from practice conditions that do not require full-body movement. For instance, older adults have been shown to be particularly focused on watching their steps to maintain their balance and avoid falling (Avineri et al., 2012). As a result, full-scale simulation devices may hamper 
795 learning in older populations due to older adults' neglecting active visual control during the

796 street-crossing phase (Woollacott \& Tang, 1997).

Further research is required to understand if training the ability to detect prospective information resulted in earlier, more precise initiations from older adults relative to the gap opening. If older adults initiated actions more tightly relative to the lead vehicle, it would then provide stronger evidence that older adults tend to wait longer to ensure they detected the appropriate perceptual variable required to allow a functional information-movement coupling. Finally, it is important to note that while older adults improved their ability to calibrate their perception of the crossability of the gaps between vehicles to the fixed walking imposed by the virtual environment, this may not transfer to the real world and their own action capabilities. Older adults tend to overestimate how quickly they can reach the opposite side of the road and may regularly need to check calibration (van Andel, Cole, \& Pepping, 2017; Zivotofsky, Eldror, Mandel, \& Rosenbloom, 2012). As the present task did not include a transfer task, further work is needed to better understand how both characteristics of the individual and the properties of the environment jointly influence the ability to learn safer road-crossing skills.

\section{Conclusions}

In conclusion, the results of the study underscore the importance of informational and feedback design for perceptual learning of affordances. In general, older adults were able to learn to improve gap-based affordance decision-making through training, as reflected in the reduced errors among all groups. Furthermore, if additional augmented cues are introduced these cues must be lawfully related to the desired information the experimenter wishes the participant to attune to. Differences between training groups showed that cross-modal cueing can educate attention both towards and away from specifying variables for action decisions. 
819 Further work is needed to understand if such effects on perceptual learning will transfer to a

820 more representative environment where an elderly pedestrian is required to couple the

821 information arising from the oncoming vehicles to their movements.

\section{Disclosure statement}

823 James Stafford and Matthew Rodger declare that they have no conflicts of interest relevant to

824 the content of this research.

825

826

827

828

829

830

831

832

833

834

835

836

837 
EDUCATING OLDER ADULTS' ATTENTION TO GAP

AFFORDANCES

839

840

841

842

843

844

845

846

847

848

849

850

851

852

853

854

855

856

857

858

859

860

861

Allard, R., Lagacé-Nadon, S., \& Faubert, J. (2013). Feature tracking and aging. Frontiers in psychology, 4, 427.

Araújo, D., Davids, K., \& Serpa, S. (2005). An ecological approach to expertise effects in decision-making in a simulated sailing regatta. Psychology of Sport and Exercise, 6(6), 671-692.

Avineri, E., Shinar, D., \& Susilo, Y. O. (2012). Pedestrians' behaviour in cross walks: the effects of fear of falling and age. Accident Analysis \& Prevention, 44(1), 30-34.

Bootsma, R. J., \& Oudejans, R. R. (1993). Visual information about time-to-collision between two objects. Journal of experimental psychology: human perception and performance, 19(5), 1041.

Brand, M. T., \& de Oliveira, R. F. (2017). Recalibration in functional perceptualmotor tasks: A systematic review. Human movement science, 56, 54-70.

Braly, A. M. (2020). Direct Learning for Time-to-Collision Judgments of Approaching Objects: The Role of Fractal 1/f Noise in Exploration (Doctoral dissertation, Rice University).

Brunswik, E. (1955). Representative design and probabilistic theory in a functional psychology. Psychological review, 62(3), 193.

Butler, A. A., Lord, S. R., \& Fitzpatrick, R. C. (2016). Perceptions of speed and risk: experimental studies of road crossing by older people. PLoS one, 11(4).

Coeugnet, S., Dommes, A., Panëels, S., Chevalier, A., Vienne, F., Dang, N. T., \& Anastassova, M. (2017). A vibrotactile wristband to help older pedestrians make safer streetcrossing decisions. Accident Analysis \& Prevention, 109, 1-9. 
Crosswalks. Transportation Research Record, 1487, 63. ideas in psychology, 36, 50-59. basketball. Revista de psicología del deporte, 18(3), 475-479. pass decisional behavior in rugby union. Human Movement Science, 30(5), 984-997. where a ball will go: the case of curved free kicks in football. Naturwissenschaften, 93(2), 97101. psychology and behaviour, 6(1), 63-80. 
Dommes, A., \& Cavallo, V. (2012). Can simulator-based training improve street-

884

885

886

887 crossing safety for elderly pedestrians?. Transportation research part F: traffic psychology and behaviour, 15(2), 206-218.

Dommes, A., Cavallo, V., Vienne, F., Aillerie, I., 2012. Age-related differences in street-crossing safety before and after training of older pedestrians. Accid. Anal. Prev. 44, $42-47$.

Dyer, J. F., Stapleton, P., \& Rodger, M. (2017). Mapping sonification for perception and action in motor skill learning. Frontiers in neuroscience, 11, 463.

Ernst, M. O., \& Banks, M. S. (2002). Humans integrate visual and haptic information in a statistically optimal fashion. Nature, 415(6870), 429-433.Farnell, A. (2010). Designing Sound. MIT Press.

Fitzpatrick, P., Carello, C., Schmidt, R. C., \& Corey, D. (1994). Haptic and visual perception of an affordance for upright posture. Ecological Psychology, 6(4), 265-287.

Franchak, J. M., Celano, E. C., \& Adolph, K. E. (2012). Perception of passage through openings depends on the size of the body in motion. Experimental brain research, 223(2), 301-310.

Geraghty, J., Holland, C., \& Rochelle, K. (2016). Examining links between cognitive markers, movement initiation and change, and pedestrian safety in older adults. Accident Analysis \& Prevention, 89, 151-159.

Gibson, E. J. (1969). Principles of perceptual learning and development. AppletonCentury Crofts, New York.

Gibson, J. J. (1979). An ecological approach to visual perception. Boston MA: 

realism: The selected essays of James J. Gibson (pp. 401-418). Hillsdale, NJ: Erlbaum on audiovisual time-to-arrival judgments. Perception \& psychophysics, 67(4), 580-594.

Perceiving and acting on complex affordances: How children and adults bicycle across two lanes of opposing traffic. Journal of experimental psychology: human perception and performance, 39(1), 23. through apertures. Journal of Experimental Psychology: Human Perception and Performance, 34(6), 1501. variables, attunement, and calibration. Journal of Experimental Psychology: Human Perception and Performance, 32(2), 443. psychology, 19(4), 321-349. the relative mass of colliding balls in globally and locally constrained task ecologies. Journal of Experimental Psychology: Human Perception and Performance, 27(5), 1019. 

relative weights of visual and auditory tau versus heuristic-based cues for time-to-contact judgments in realistic, familiar scenes by older and younger adults. Attention, perception \& psychophysics, 79(3), 929. movement in relation to age and muscle morphology. Journal of Applied Physiology, 46(3), 451-456.

Lee, D. N. (1976). A theory of visual control of braking based on information about time-to-collision. Perception, 5(4), 437-459. 4), $221-250$ optics. Nature, 293(5830), 293-294. Psychology, 35(2), 333-346. 
EDUCATING OLDER ADULTS' ATTENTION TO GAP

AFFORDANCES

950

951

952

953

954

955

956

957

958

959

960

961

962

963

964

965

966

967

968

969

970

971

972

Lobjois, R., \& Cavallo, V. (2007). Age-related differences in street-crossing

decisions: The effects of vehicle speed and time constraints on gap selection in an estimation task. Accident Analysis \& Prevention, 39(5), 934-943.

Lynch, S. D., Olivier, A. H., Bideau, B., \& Kulpa, R. (2019). Detection of deceptive motions in rugby from visual motion cues. PloS one, 14(9), e0220878.

Oudejans, R. R., Michaels, C. F., Bakker, F. C., \& Dolne, M. A. (1996). The

relevance of action in perceiving affordances: Perception of catchableness of fly

balls. Journal of Experimental Psychology: Human Perception and Performance, 22(4), 879..

Oudejans, R. R., Michaels, C. F., Van Dort, B., \& Frissen, E. J. (1996). To cross or not to cross: The effect of locomotion on street-crossing behavior. Ecological psychology, 8(3), 259-267.

Owsley, C. (2011). Aging and vision. Vision research, 51(13), 1610-1622.

Oxley, J. A., Ihsen, E., Fildes, B. N., Charlton, J. L., \& Day, R. H. (2005). Crossing roads safely: an experimental study of age differences in gap selection by pedestrians. Accident Analysis \& Prevention, 37(5), 962-971.

Park, J. H., Shea, C. H., \& Wright, D. L. (2000). Reduced-frequency concurrent and terminal feedback: a test of the guidance hypothesis. Journal of motor behavior, 32(3), $287-$ 296.

Peiffer, A. M., Mozolic, J. L., Hugenschmidt, C. E., \& Laurienti, P. J. (2007). Agerelated multisensory enhancement in a simple audiovisual detection task. Neuroreport, 18(10), 1077-1081.

Petzoldt, T. (2014). On the relationship between pedestrian gap acceptance and time to arrival estimates. Accident Analysis \& Prevention, 72, 127-133. 
Poulter, D. R., \& Wann, J. P. (2013). Errors in motion processing amongst older drivers may increase accident risk. Accident Analysis \& Prevention, 57, 150-156. for auditory perception. Journal of Cognitive Psychology, 29(1), 65-87. audiovisual facilitation of object motion within self-motion. Multisensory research, 31(3-4), $251-272$.

Runeson, S., \& Andersson, I. E. (2007). Achievement of specificational information usage with true and false feedback in learning a visual relative-mass discrimination task. Journal of Experimental Psychology: Human Perception and Performance, 33(1), 163. specifying time-to-contact. Ecological Psychology, 3(3), 253-261. perception of gap affordances: impact of standardized action capabilities on road-crossing judgements. Accident Analysis \& Prevention, 129, 21-29. perceptual-motor calibration to changes in action capabilities. Human movement science, 51, 59-71.van der Meer, A. L., van der Weel, F. R., \& Lee, D. N. (1994). Prospective control in 
EDUCATING OLDER ADULTS' ATTENTION TO GAP

AFFORDANCES

995

996

997

998

999

1000

1001

1002

1003

1004

1005

1006

1007

1008

1009

1010

1011

1012

1013

1014

1015

van Doorn, H., van der Kamp, J., de Wit, M., \& Savelsbergh, G. J. (2009). Another

look at the Müller-Lyer illusion: Different gaze patterns in vision for action and perception. Neuropsychologia, 47(3), 804-812.

Warren Jr, W. H., \& Whang, S. (1987). Visual guidance of walking through apertures: body-scaled information for affordances. Journal of experimental psychology: human perception and performance, 13(3), 371.

Watson, G., Brault, S., Kulpa, R., Bideau, B., Butterfield, J., \& Craig, C. (2011). Judging the 'passability' of dynamic gaps in a virtual rugby environment. Human Movement Science, 30(5), 942-956.

Withagen, R. (2004). The pickup of nonspecifying variables does not entail indirect perception. Ecological Psychology, 16(3), 237-253.

Withagen, R., \& Caljouw, S. R. (2011). Aging affects attunement in perceiving length by dynamic touch. Attention, Perception, \& Psychophysics, 73(4), 1216-1226.

Withagen, R., \& Michaels, C. F. (2005). The role of feedback information for calibration and attunement in perceiving length by dynamic touch. Journal of Experimental Psychology: Human Perception and Performance, 31(6), 1379.

Withagen, R., \& Van Wermeskerken, M. (2009). Individual differences in learning to perceive length by dynamic touch: Evidence for variation in perceptual learning capacities. Perception \& Psychophysics, 71(1), 64-75.

Woollacott, M. H., \& Pei-Fang, T. (1997). Balance control during walking in the older adult: research and its implications. Physical therapy, 77(6), 646. 
Zivotofsky, A. Z., Eldror, E., Mandel, R., \& Rosenbloom, T. (2012). Misjudging their 607.

\section{Appendix 1}

1022

1023

Table 1. Combinations of the different conditions for virtual cars' speed, distance, the resulting time-to-arrival, and the time 1024 differentials.

1025

\begin{tabular}{lllll}
\hline \hline $\begin{array}{l}\text { Speed } \\
(\mathrm{M} / \mathrm{S})\end{array}$ & $\begin{array}{l}\text { Starting } \\
\text { Distance }(\mathrm{M})\end{array}$ & $\begin{array}{l}\text { Distance At Gap } \\
\text { Opening }(\mathrm{M})\end{array}$ & $\begin{array}{l}\text { Time-to-arrival } \\
\text { (Speed/Distance) }\end{array}$ & Time Differential (s) \\
\hline \hline & & & & \\
9.44 & 160 & 23.78 & 2.52 & -1.43 \\
10 & 164 & 27.6 & 2.76 & -1.19 \\
11.67 & 175 & 33.26 & 2.85 & -1.1 \\
7.22 & 146 & 21.95 & 3.04 & -0.91 \\
11.11 & 171 & 36.77 & 3.31 & -0.63 \\
12.78 & 182 & 42.56 & 3.33 & -0.61 \\
13.33 & 185 & 48.12 & 3.61 & -0.34 \\
12.22 & 178 & 44.36 & 3.63 & -0.32 \\
5 & 157 & 18.3 & 3.66 & -0.29 \\
7.78 & 150 & 31.35 & 4.03 & 0.09 \\
8.33 & 153 & 35.07 & 4.21 & 0.26 \\
6.11 & 139 & 25.72 & 4.21 & 0.27 \\
3.89 & 125 & 16.42 & 4.22 & 0.28 \\
10.56 & 167 & 46.25 & 4.38 & 0.45 \\
6.67 & 142 & 29.41 & 4.41 & 0.49 \\
4.44 & 128 & 20.2 & 4.55 & 0.59 \\
8.89 & 132 & 40.72 & 4.58 & 0.64 \\
5.56 & 135 & 38.86 & 6.99 & 3.05 \\
\hline
\end{tabular}

1026 


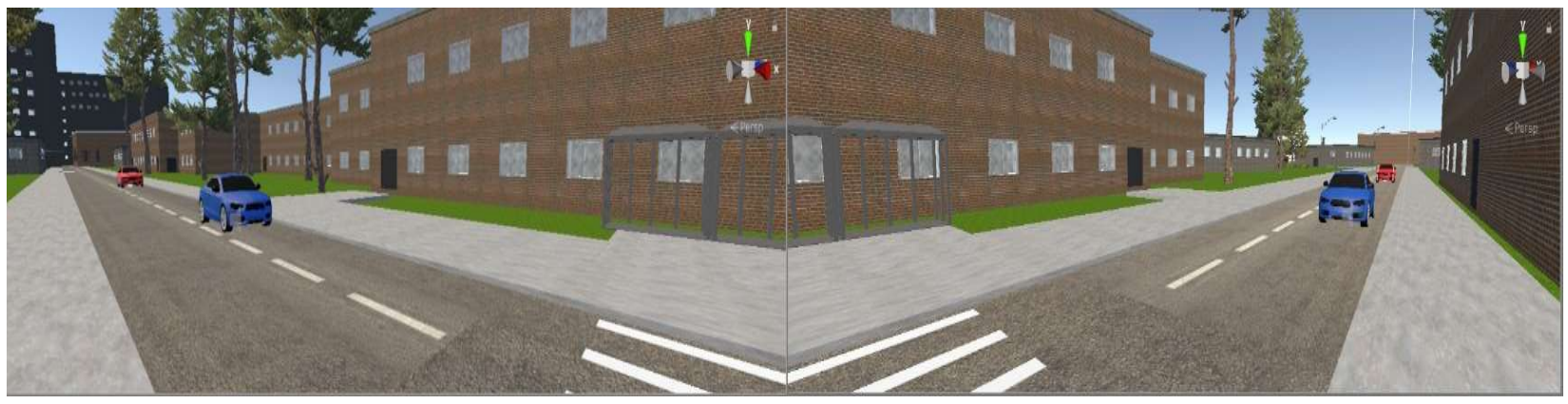

1037

Fig. 1. Screenshot from the pedestrian's perspective while looking either left or right of the vehicles approach in

1038 both the near and far lane.

1039

1040

1041 


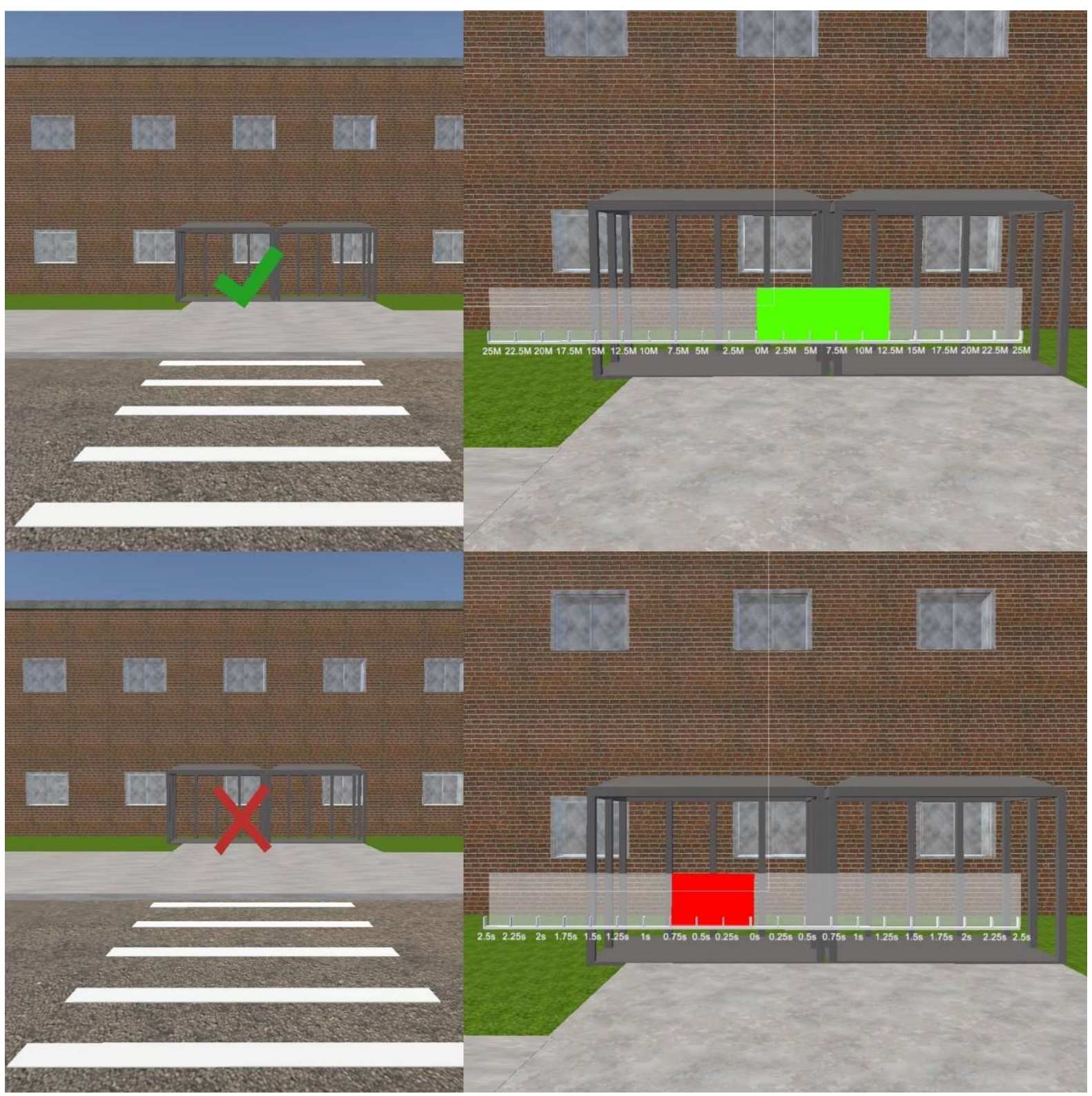

Fig. 2. The KR feedback received in both training phases. If participants rejected an opportunity that afforded safe passage, they received a red ' $\mathrm{X}$ '. If participants rejected an opportunity that afforded collision, they received a green tick. If a participant crossed they received a safety margin gauge. The non-specifying group received feedback in the form of distance of the approaching vehicle once they reached the opposite curb. The specifying group received a gauge in seconds to spare. The bar was green if the participant safely made it across without collision and red if they collided with the approaching vehicle 


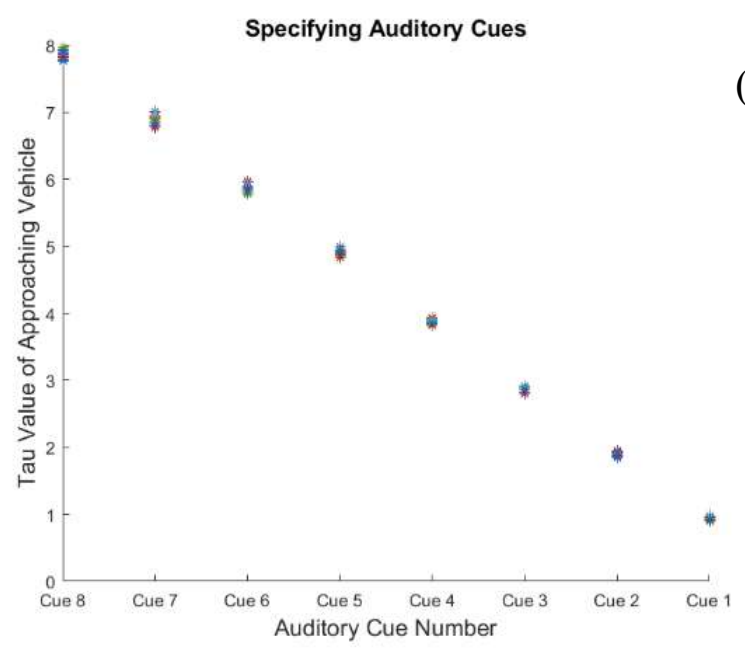

(a)

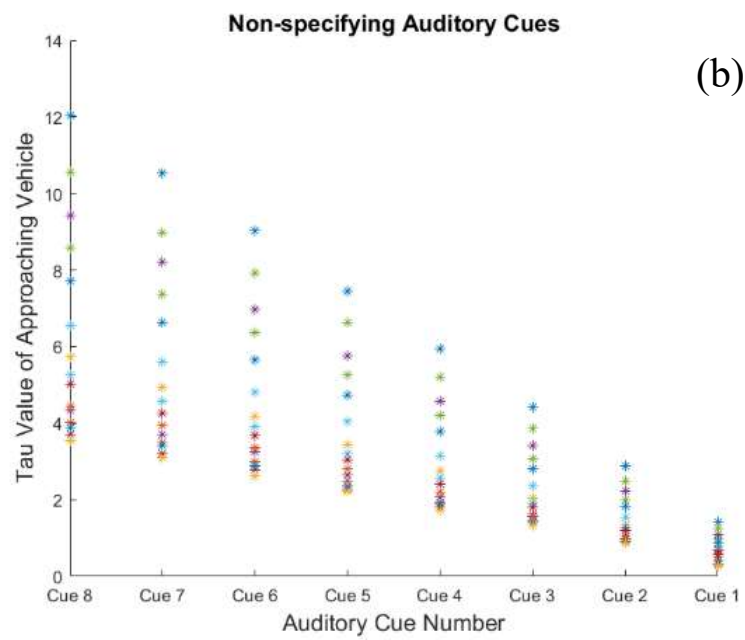

Fig. 3. Optical tau values for each auditory cue trigger used in the experiment as the vehicle approached the

1053

1054

1055

1056

1057

1058

1059

1060 participant. Different coloured circles indicate different speed/distance combinations. Figure 3a shows a near-identical linear relationship across trials between the onsets of the auditory cues and the tau value of the approaching vehicle (as calculated from the ratio of the car's optical angle and this angle's rate-of-change in the VR presentations of these conditions).

Conversely, figure $3 \mathrm{~b}$ shows variable linear relationships across conditions between the auditory cue onsets and the concurrent tau values of the approaching vehicle.

\section{Table 2}

Summary of means (SDs) for each cue group including $\%$ of gaps accepted, $\%$ of correct responses, $\%$ of unsafe errors, and $\%$ of safe errors across the three different test phases in the road-crossing task

\begin{tabular}{|c|c|c|c|c|}
\hline \multicolumn{2}{|l|}{ Cue Group } & $\%$ Gaps & $\%$ Unsafe & $\%$ Safe \\
\hline \multirow{3}{*}{ Control } & Pre-test & $4017 \%(161)$ & $1351 \%(97)$ & $2656 \%(1035)$ \\
\hline & Post-test & $45.94 \%(18.8)$ & $13.81 \%(9.5)$ & $17.88 \%(11.6)$ \\
\hline & Retention & $43.45 \%(18.7)$ & $11.11 \%(10.2)$ & $21.79 \%(9.1)$ \\
\hline \multirow[t]{3}{*}{ Non-specifying } & Pre-test & $39.45 \%(11.5)$ & $13.45 \%(6.2 \%)$ & $27.83 \%(10.6)$ \\
\hline & Post-test & $45.3 \%(12.8)$ & $14.87 \%(2.8)$ & $20.27 \%(10.5)$ \\
\hline & Retention & $43.87 \%(7.72)$ & $12.5 \%(7.1)$ & $21.65 \%(8.9)$ \\
\hline \multirow[t]{3}{*}{ Specifying } & Pre-test & $39.6 \%(17.1)$ & $13.25 \%(7.1)$ & $26.92 \%(10.7)$ \\
\hline & Post-test & $42.3 \%(10.3)$ & $10.22 \%(5.2)$ & $16.46 \%(8.01)$ \\
\hline & Retention & $45.6 \%(12.6)$ & $14.97 \%(6.2)$ & $16.68 \%(9.4)$ \\
\hline
\end{tabular}



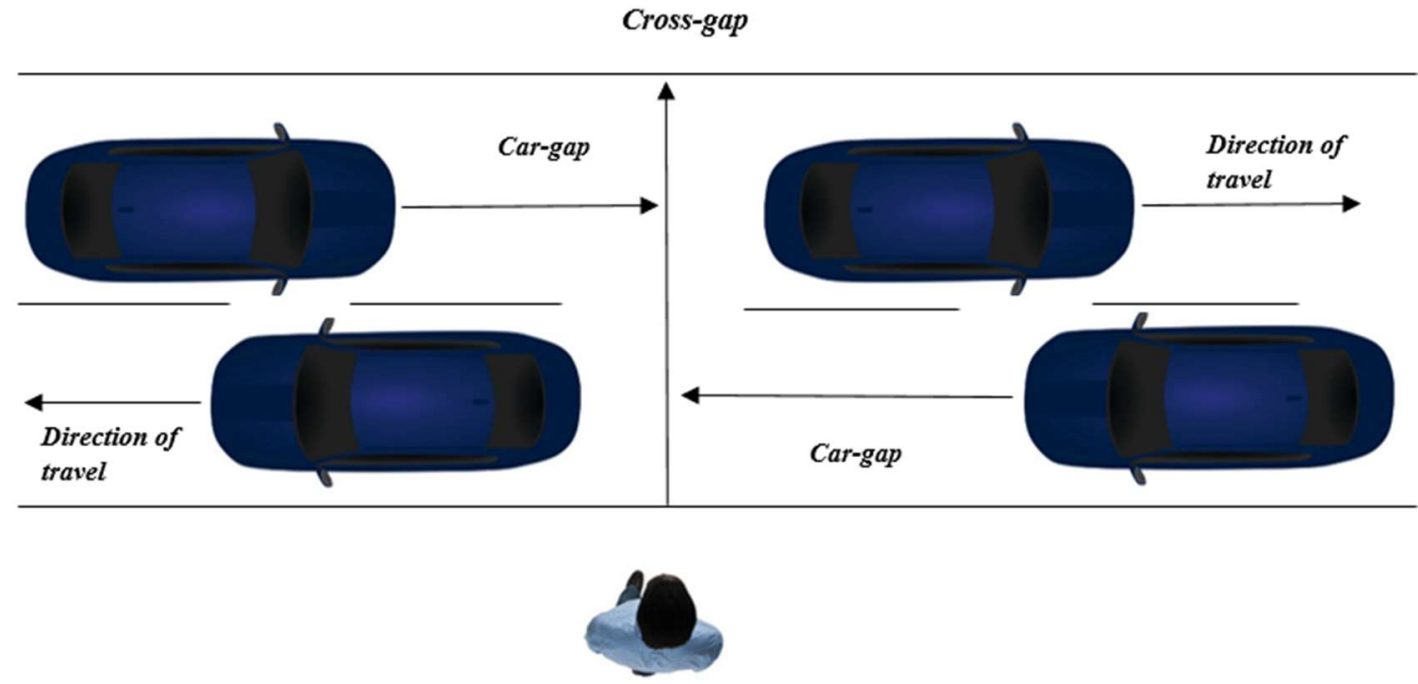

Fig. 4. A schematic diagram showing the axes of movement of the two lanes of cars and the gaps between them. The time difference value is represented by the Car-gap between the participant and the second vehicle at the moment the rear bumper of the first vehicle passes the participant.

Table 3

Output of the logistic functions for each cue group including variance explained for candidate information sources: timedifferential (along with its critical value and slope) and gap magnitude $\left(\mathrm{R}^{2)}\right.$ across the three different test phases in the roadcrossing task

\begin{tabular}{|c|c|c|c|c|c|}
\hline Cue Group & & $\begin{array}{l}\text { Time } \\
\text { difference } \mathrm{R}^{2} \\
\end{array}$ & Critical Value & Slope & $\begin{array}{l}\text { Gap magnitude } \\
\mathrm{R}^{2}\end{array}$ \\
\hline \multirow[t]{3}{*}{ Control } & Pre-test & 0.592 & 0.32 & 1 & 0.48 \\
\hline & Post-test & 0.808 & 0.01 & 0.73 & 0.005 \\
\hline & Retention & 0.814 & 0.07 & 0.61 & 0.06 \\
\hline \multirow{3}{*}{$\begin{array}{l}\text { Non- } \\
\text { specifying }\end{array}$} & Pre-test & 0.557 & 0.37 & 1.01 & 0.508 \\
\hline & Post-test & 0.472 & 0.08 & 0.87 & 0.369 \\
\hline & Retention & 0.581 & 0.07 & 0.76 & 0.268 \\
\hline \multirow[t]{3}{*}{ Specifying } & Pre-test & 0.64 & 0.35 & 1 & 0.362 \\
\hline & Post-test & 0.812 & 0.08 & 0.56 & 0.004 \\
\hline & Retention & 0.749 & -0.1 & 0.6 & 0.06 \\
\hline
\end{tabular}


Pre-test
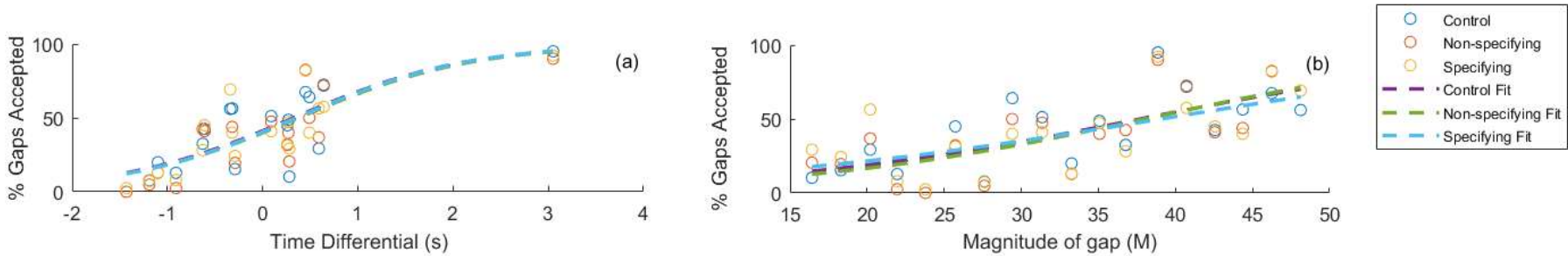

Post-test
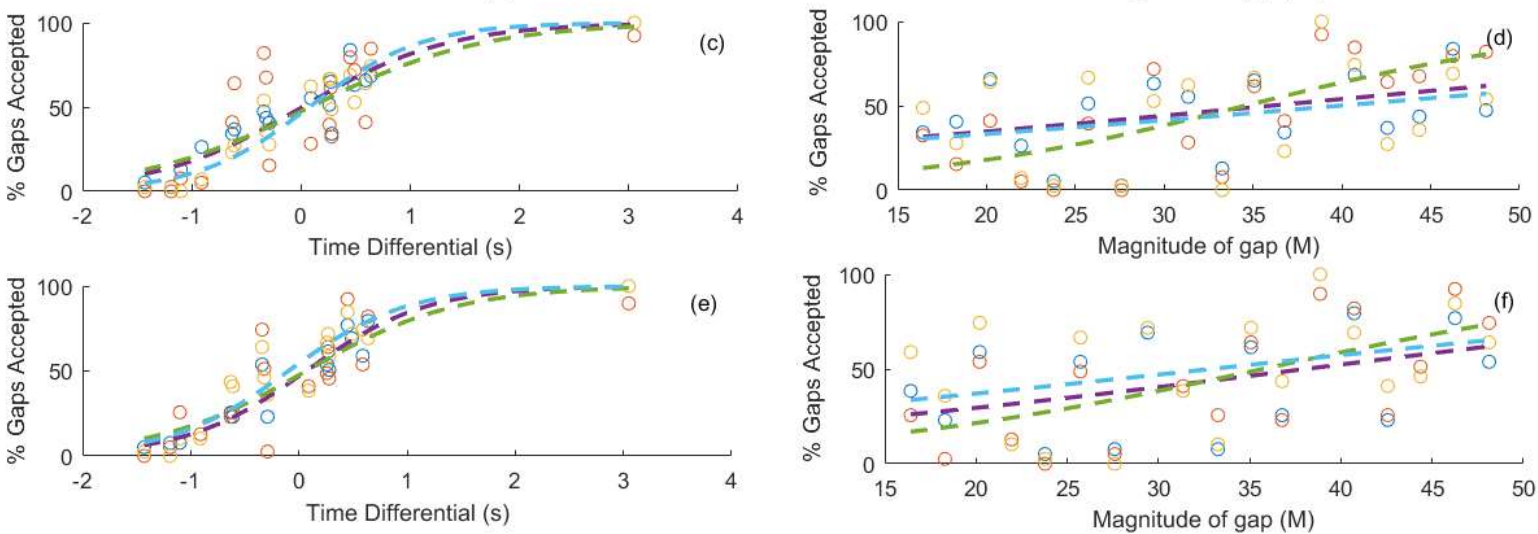

1081

1082

1083

1084

1085

Fig. 5. Figures showing the logistic functions for the $\%$ of gaps accepted for the three experimental groups during the pretest phase, post-test phase, and the retention phase. For each phase, a logistic function was fitted for the candidate informational variables: time differential and magnitude of gap. Gap closure-rate was excluded due to low amounts of variance explained across all three phases (see supplementary materials).

1086

1087

1088

1089

1090

1091

1092

1093 
EDUCATING OLDER ADULTS' ATTENTION TO GAP

AFFORDANCES

\section{Supplementary Materials}

1096

1097

1098

1099

1100

1101

1102

1103

1104

1105

1106

1107

1108

1109

1110

1111

1112

1113

1114

1115

Analysis of gap closure-rate

***INSERT FIGURE 6***

During the pre-test phase, the rate of closure of the gap (at the group level) only accounted for $3.45 \%$ of the variance in the specifying group, $0.01 \%$ of the variance in the non-specifying group, and $0.01 \%$ of the variance in the control group suggesting that gapclosure alone did not inform judgements before training. Similarly, during the post-test phase, the rate of closure of the gap only accounted for $0.05 \%$ of the variance in the specifying, nonspecifying, and control group. This indicates that the auditory cues (specifying and nonspecifying) did not educate participant's attention towards gap-closure information. Finally, Figure $6 \mathrm{c}$ shows the retention phase where gap closure-rate accounted for $0.02 \%$ of the variance in the specifying group, $0.03 \%$ of the variance in the non-specifying group, and $0.01 \%$ of the variance in the control group.

For completeness, we also analysed the logistic gap closure/decision rate fits for each participant using ANCOVA (pre-test as covariate). No effect of training group was found for post-test $\left(F(3,39)=.759, p=.476, \eta^{2}=.04\right)$ or retention $\left(F(3,39)=.940, p=.40, \eta^{2}=.05\right)$. In summary, participants at no point during the experiment were basing their responses on how fast the gap was closing. The fact that gap closure-rate was not informing action-decisions is particularly interesting for the non-specifying group as the auditory cues presented to this group tracked both speed and distance information. These findings are in line with previous research showing older adults often fail to account for vehicle speed and instead opt for distance-based heuristics (Oxley et al., 2005; Lobjois \& Cavallo, 2007). As a result, the nonspecifying group during training were more likely to tune into the individual sound onsets 
EDUCATING OLDER ADULTS' ATTENTION TO GAP

AFFORDANCES

1118 (specifying gap magnitude) rather than the difference in interval durations of the sounds

1119 (specifying gap closure-rate).

1120

Did the auditory cues cause a guidance effect during training?

1121

******INSERT FIGURE 7*******

Although the primary aim of the present experiment was to understand if older adult's

1123

1124

1125

1126

1127

1128

1129

1130

1131

1132

1133 attention could be educated towards and/or away from gap-specifying information, it is useful to understand if the participants were relying too heavily on the auditory cues during training to inform road-crossing judgements. Figure 7 demonstrates how the average percentage of correct decisions did not drastically change during the two training phases, indicating that both the specifying and non-specifying group were not substantially improving in accuracy when the auditory cues were presented during training. The guidance hypothesis predicts that learners will show a significant decline in performance when cues are removed, which these results do not indicate for the present study for any of the three groups. This suggests that the auditory cues presented in this experiment are do not lead to older adults' perceptual learning being reliant on the presence of augmented cues.

***INSERT FIGURE 8***

Regarding which variables were used to select gaps during training, Figure 8 shows that both the specifying group and the control group gradually improved in their ability to tune into the time differential over the course of the two training phases which was maintained in the post-test phase. In contrast, the non-specifying group, initially improved in their ability to tune into the time differential value in the first training phase. However, this noticeably reduced during the second training phase implying that the non-specifying auditory cues took longer to influence learning. Similar to the control and specifying group, the non-specifying group maintained their variable usage from the second training phase to the post-test phase. 
EDUCATING OLDER ADULTS' ATTENTION TO GAP

AFFORDANCES

52

1142 The fact that all groups did not dramatically change variable usage between the second

1143 training phase and the post-test phase suggests that the auditory cues used in the present

1144 experiment were able to circumvent any guidance effects in information usage also.

1145

1146

1147

1148

1149

1150

1151

1152

1153

1154

1155

1156

1157

1158

1159

1160

1161 


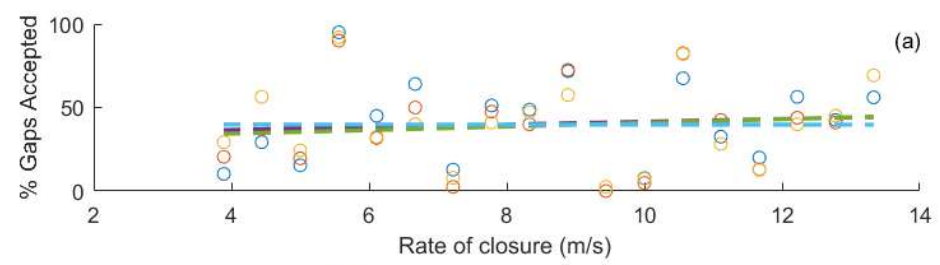

\begin{tabular}{|c|c|}
\hline 0 & Control \\
\hline 0 & Non-specifying \\
\hline 0 & Specifying \\
\hline-- & - Control fit \\
\hline & $\begin{array}{l}\text { Non-specifying fit } \\
\text { Specifying fit }\end{array}$ \\
\hline
\end{tabular}

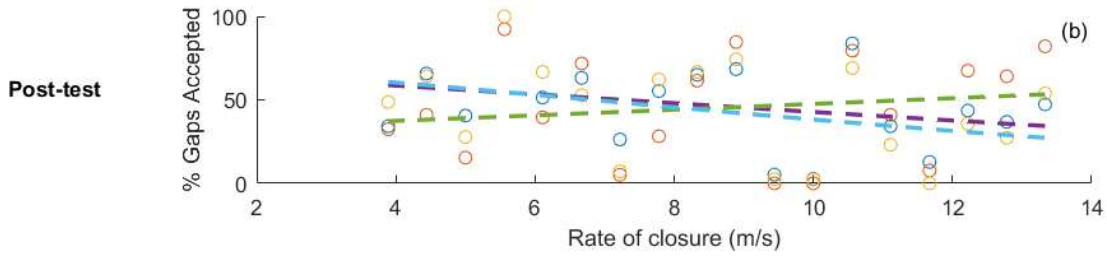

(b)

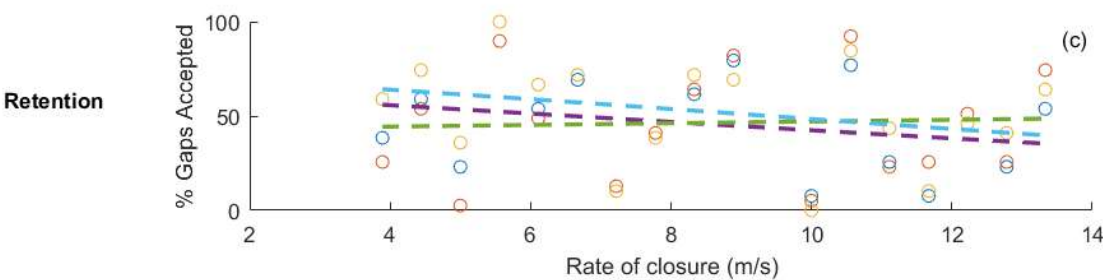

(c)

Fig. 6. Logistic functions for the $\%$ of gaps accepted based on gap closure-rate for the three experimental groups during the pre-test phase, post-test phase, and the retention phase.

1166

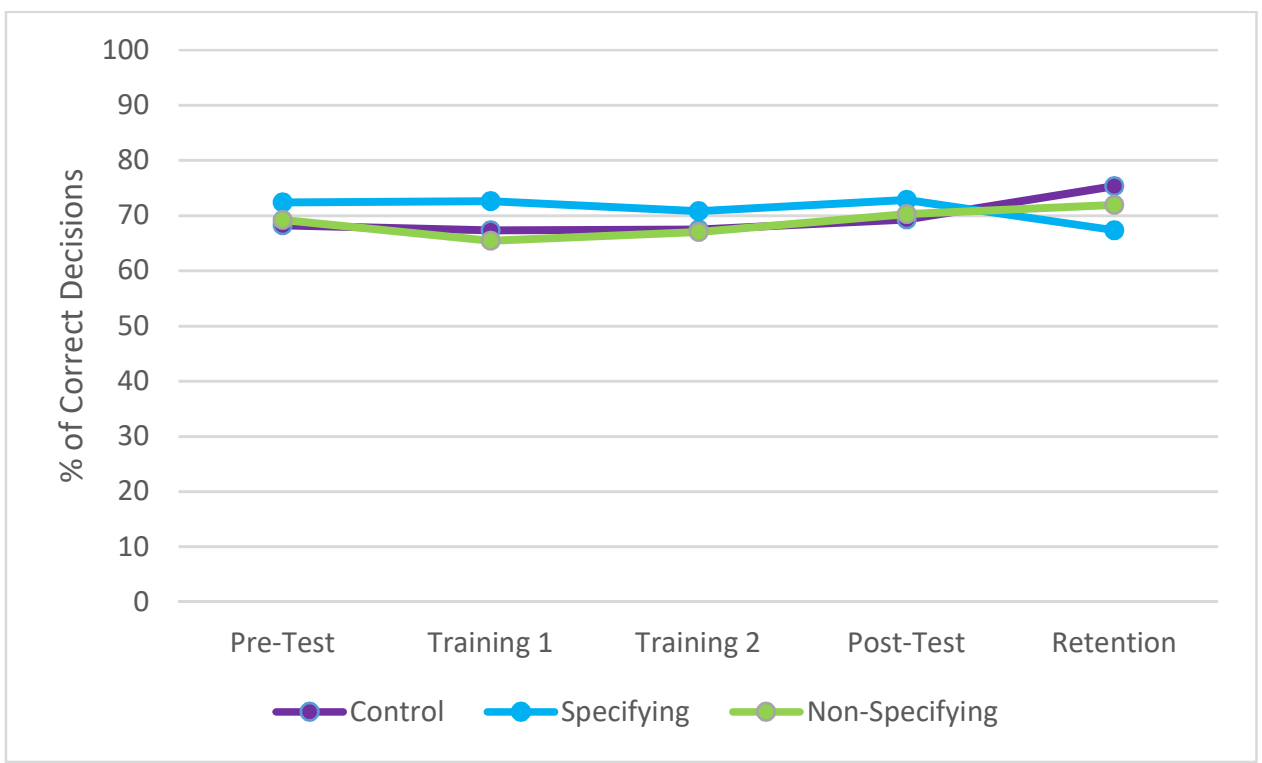

Fig. 7. Average $\%$ of correct decisions for each group (i.e. accepting the gap when it was large enough and rejecting it when 
EDUCATING OLDER ADULTS’'ATTENTION TO GAP

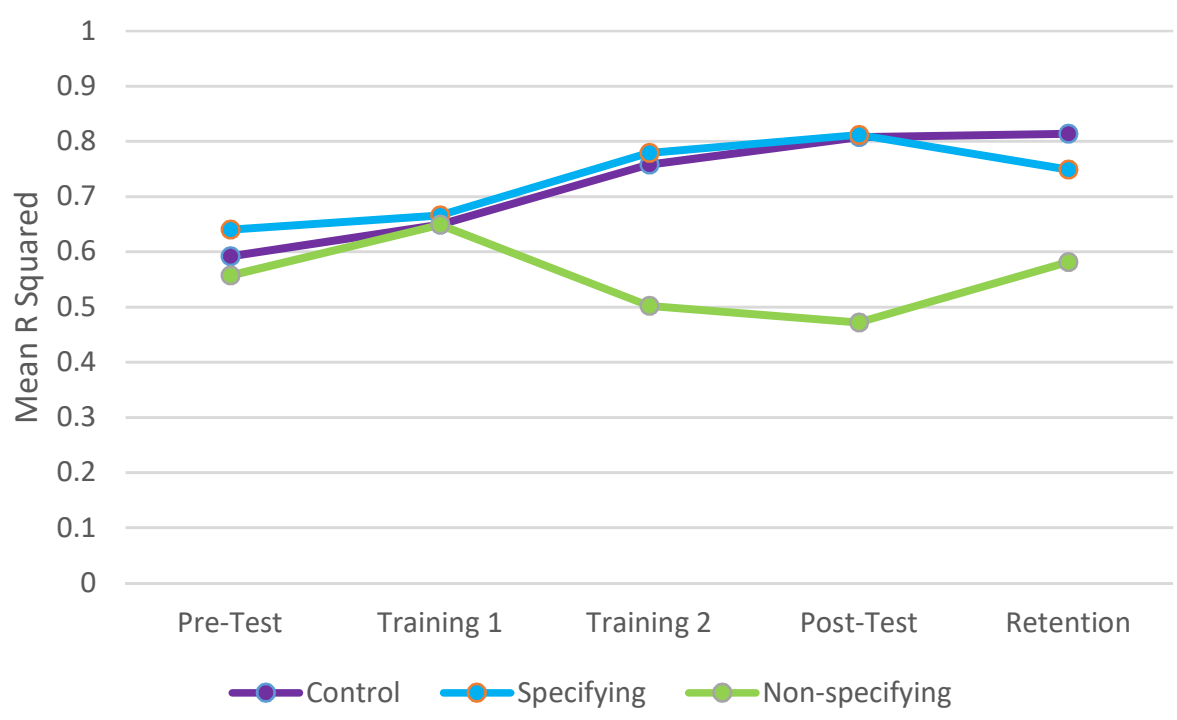

Fig. 8. Mean $R$-squared values of logistic functions fitted between time-differentials and yes/no judgement rates across test phases. These values represent the extent each group was using the time differential variable to inform gap selection. 